\title{
Macrophage migration inhibitory factor regulates cyst growth in ADPKD
}

Macrophage migration inhibitory factor (MIF) has roles in the recruitment of immune cells to sites of inflammation and in the induction of inflammatory mediators, including tumour necrosis factor (TNF). Recent data from Xiaogang $\mathrm{Li}$ and colleagues suggest that MIF also has an important role in the pathogenesis of autosomal dominant polycystic kidney disease (ADPKD).

"Our previous finding that TNF promotes cystogenesis initiated a novel research direction regarding the role of renal inflammation in PKD," says Li. "Macrophages were subsequently identified in the pericystic and interstitial regions of cystic kidneys, but the mechanisms that promoted their recruitment to these sites and the origin of TNF in cyst fluid were unknown. In our new study we investigated the role of MIF in macrophage recruitment and TNF production in kidney cysts."

The researchers found that levels of MIF were increased in murine renal epithelial cells with $P k d 1$ mutations compared with wild-type controls, and in the cyst fluid and urine of patients with ADPKD compared with normal kidney and urine samples. In mouse models of ADPKD with $P k d 1$ mutations, inhibition or knockout of MIF delayed cyst growth and reduced renal macrophage accumulation. MIF inhibition or knockdown also decreased glycolysis, glucose uptake, ATP production and activation of ERK, mTOR and AMPK signalling pathways in cystic renal epithelial cells. MIF induced TNF expression in renal epithelial cells and vice versa, suggesting that a positive feedback loop between these cytokines might contribute to cyst development.

"MIF not only regulates the recruitment of macrophages to cystic kidneys, but also has a critical upstream role in the regulation of $\mathrm{PKD}$-associated signalling pathways, glucose uptake and glycolysis in renal epithelial cells," explains Li. "Our findings provide evidence that the Warburg effect-that is, reprogramming of glucose metabolism to use aerobic glycolysisobserved in cancer and PKD might be mediated by MIF."

As MIF knockouts have no overt phenotype, the researchers suggest that this cytokine would be a good therapeutic target and has translational potential for ameliorating the progression of ADPKD. "Our study is highly significant as it enables a better understanding of the mechanisms of renal cyst formation and identifies a novel therapeutic target-and potential urinary biomarker-for ADPKD," concludes Li.

Ellen F. Carney

Original article Chen, L. et al. Macrophage migration inhibitory factor promotes cyst growth in polycystic kidney disease. J. Clin. Invest. doi:10.1172/JCI80467 